\title{
SANS Study of Protein Adsorption on Nanoparticles
}

\author{
Sugam Kumar ${ }^{1}$, V.K. Aswal ${ }^{1 *}$ and J. Kohlbrecher ${ }^{2}$ \\ ${ }^{1}$ Solid State Physics Division, Bhabha Atomic Research Centre, Trombay, Mumbai 400 085, INDIA \\ ${ }^{2}$ Laboratory for Neutron Scattering, ETH Zurich \& Paul Scherrer Institute, CH-5232 PSI Villigen, Switzerland \\ "E-mail:vkaswal@barc.gov.in
}

\begin{abstract}
Adsorption of lysozyme protein on silica nanoparticle has been studied using small-angle neutron scattering (SANS) at $\mathrm{pH}$ 7. The measurements were carried out on fixed concentration $(1 \mathrm{wt} \%)$ of nanoparticles and varying concentration of protein in the range 0 to $2 \mathrm{wt} \%$. It has been found that the protein is adsorbed on the nanoparticle surface at very low protein concentrations whereas strong electrostatic interaction of lysozyme with silica nanoparticles at higher protein concentrations leads to the aggregation of nanoparticles. The adsorption is found to be increased with increase in the particle size and the aggregation is determined to be fractal structure.
\end{abstract}

Keywords: Lysozyme protein, silica nanoparticles, adsorption, aggregation, SANS.

PACS: 81.07.-b, 87.14.Df, 83.85.Hf

\section{INTRODUCTION}

Nanoparticles are gaining importance in the emerging field of nanobiotechnology (targeted drug delivery, controlling enzymatic behavior etc.) because of their sizes comparable to that of living cells due to which these particles can gain access to and operate within the cell [1]. It is well known that in physiological environment the material is immediately covered by proteins. This phenomenon enables nanoparticles to be used as drug carrier because cellular response to the material deals with the adsorbed biological layer instead of the materials itself. Study of protein interaction with nanoparticles is a prerequisite for all these applications. The adsorption of protein on nanoparticle surface and the characteristics of the resultant structure depend on the properties of nanoparticles. Also the protein adsorption on nanoparticle surface may lead to some structural transformation affecting the entire molecules [2]. The present study examines the interaction of lysozyme protein with silica nanoparticles using small-angle neutron scattering (SANS). SANS is a well established technique for such multi-component systems.

\section{EXPERIMENTAL}

The Electrostatically stabilized colloidal suspension of silica nanoparticles and lysozyme protein were purchased from Sigma-Aldrich. The samples were prepared by dissolving weighted amount of silica nanoparticles and protein in phosphate buffer prepared in $\mathrm{D}_{2} \mathrm{O}\left(\mathrm{pH}\right.$ 7). Use of $\mathrm{D}_{2} \mathrm{O}$ instead of $\mathrm{H}_{2} \mathrm{O}$ provides better contrast for hydrogenous systems and also low incoherent background. Small-angle neutron scattering measurements were carried out at the SANS-I facility, Swiss Spallation Neutron Source SINQ, Paul Scherrer Institute, Switzerland [3]. The wavelength of neutron beam used was $6 \AA$. Data were collected at two samples to detector distances 2 and $8 \mathrm{~m}$ to cover a wave vector transfer $(\mathrm{Q})$ range of 0.008 to $0.33 \AA^{-1}$. The scattered neutrons were detected using two-dimensional $96 \mathrm{~cm} \times 96 \mathrm{~cm}$ detector. All the data were corrected and normalized to absolute scale using standard procedure.

\section{SANS ANALYSIS}

In SANS experiments, one measures the coherent differential scattering cross section per unit volume $(\mathrm{d} \Sigma / \mathrm{d} \Omega)$ as a function of $\mathrm{Q}$ and is given by [4]

$$
\frac{d \Sigma}{d \Omega}(Q)=n V^{2}\left(\rho_{p}-\rho_{s}\right)^{2} P(Q) S(Q)+B
$$

where, $n$ is number density of particles and $V$ is particle volume. $\rho_{p}$ and $\rho_{s}$ are scattering length densities of particle and solvent, respectively. $P(Q)$ is intraparticle structure factor and depends on shape and size of the particle. $S(Q)$ is interparticle structure factor and is unity for diluted system. B is a constant term representing incoherent background. 


\section{RESULTS AND DISCUSSION}

Fig. 1 shows the SANS data of 1 wt $\%$ silica nanoparticles mixed with low concentration of lysozyme protein $(0-0.1 \mathrm{wt} \%)$. The pure data of silica shows a monotonically decreasing scattering profile governed by $\mathrm{P}(\mathrm{Q})$ only since at this concentration $\mathrm{S}(\mathrm{Q}) \sim 1.0$. The silica particles are characterized by polydispersed spheres of mean radius $8.0 \mathrm{~nm}$ with a polydispersity 0.15 . The semi-axes of prolate ellipsoidal lysozyme molecules are found to be $2.2 \mathrm{~nm} \times 1.45 \mathrm{~nm} \times 1.45 \mathrm{~nm}$ [5]. It is seen that there is scattering buildup in the low $Q$ region indicating adsorption of protein on nanoparticle that leads to aggregation of particles [6].

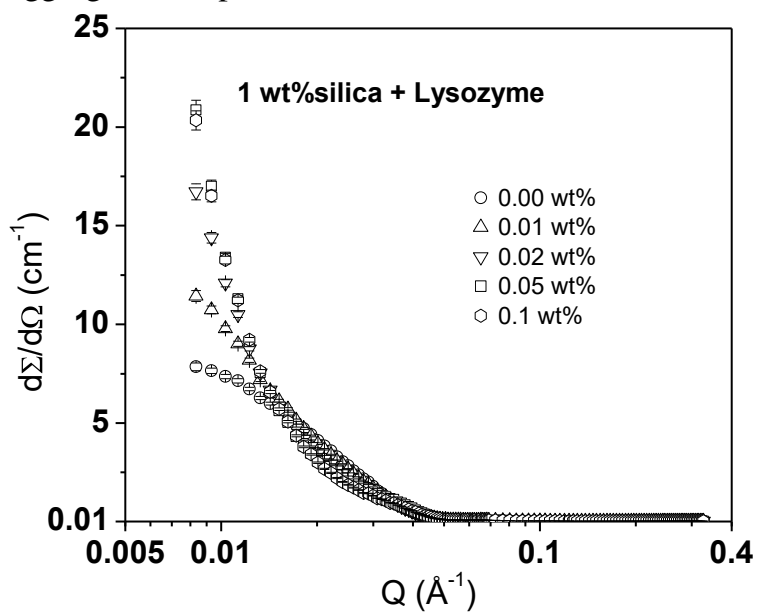

FIGURE 1. SANS data for $1 \mathrm{wt} \%$ of silica with varying (low) concentration of Lysozyme.

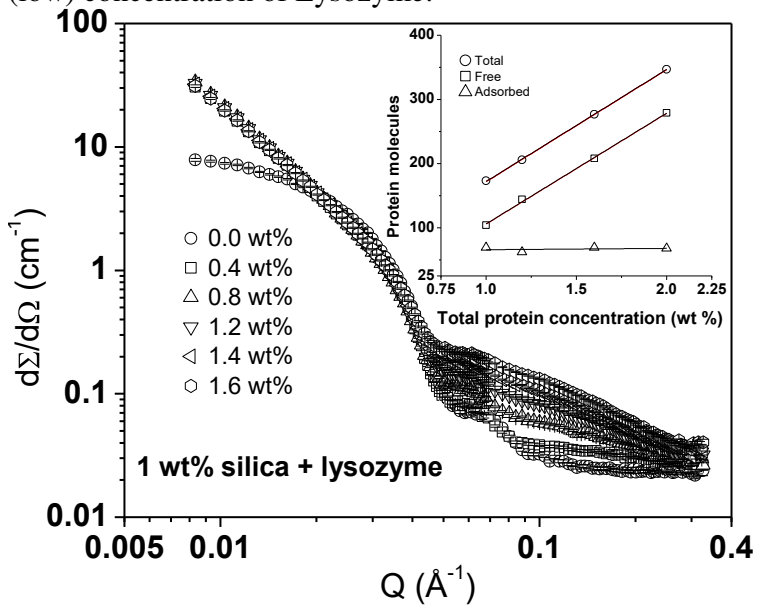

FIGURE 2. SANS data for $1 \mathrm{wt} \%$ of silica with varying (high) concentration of Lysozyme. Inset shows numbers of different protein molecules (total, free and adsorbed) in these systems.

SANS data (as shown in Fig. 2.) of $1 \mathrm{wt} \%$ silica at higher lysozyme concentrations show a large and linear dependence of scattering on log-log scale suggesting the fractal structure of aggregates of silica particles mediated by the adsorbed oppositely charged proteins on the silica particles. The fractal dimension of aggregate structure has a value 2.5 [7]. The scattering buildup in the higher $\mathrm{Q}$ range with increase in protein concentrations is observed because of existence free proteins along with silica aggregates in these systems. The calculated numbers of different protein molecules (total, free and adsorbed) are shown in the inset of Fig. 2. The maximum value of adsorbed protein molecules is found to be about 63 corresponding to $0.35 \mathrm{wt} \%$ of lysozyme protein solution.

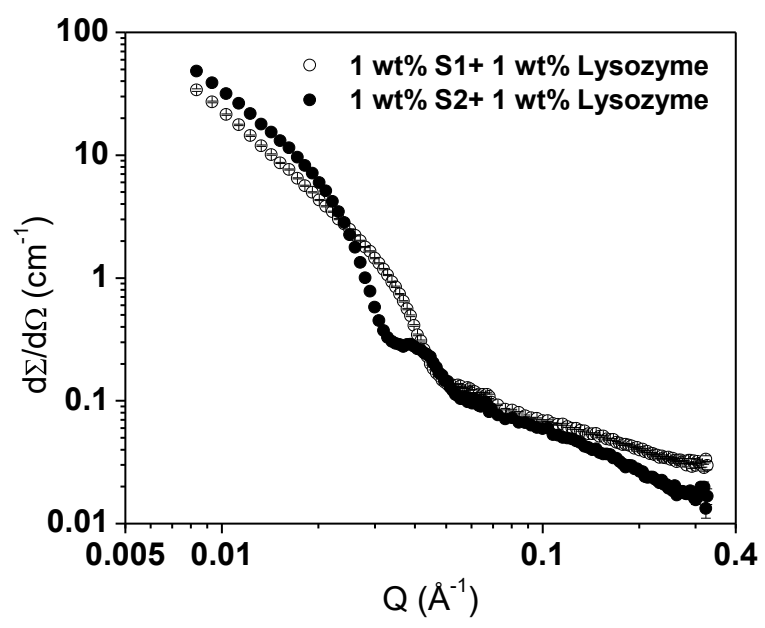

FIGURE 3. SANS data for $1 \mathrm{wt} \%$ of silica of particle sizes $8 \mathrm{~nm}$ and $11 \mathrm{~nm}$ with $1 \mathrm{wt} \%$ lysozyme.

SANS data of $1 \mathrm{wt} \%$ lysozyme with $1 \mathrm{wt} \%$ silica nanoparticles of two sizes $8 \mathrm{~nm}(\mathrm{~S} 1)$ and $11 \mathrm{~nm}(\mathrm{~S} 2)$ are shown in Fig. 3. Both the systems show fractal aggregation of silica particles. The fractal dimension remains same irrespective of the size of the particle. On the other hand adsorption increases from 63 to 171 protein molecules per particle for larger sized silica particles. This change can be understood in terms of surface dependent enhanced adsorption of protein molecules for larger particles [6].

\section{REFERENCES}

1. I. Lynch and K. A Dawson, Nano Today 3, 40 (2008).

2. I. Lynch, T.Cedervall, M. Lundqvist, C.C Lago, S. Linse and K. A Dawson, Adv. Colloid Interface Sci. 134-134, 167 (2007).

3. J. Kohlbrecher and W. Wagner, J. Appl. Cryst. 33, 804 (2000).

4. V.K. Aswal and P.S Goyal, Phys. Rev. E 79, 947 (2000).

5. S. Chodankar and V.K Aswal, Phys. Rev. E 72, 041931 (2005)

6. A.A Vertegel, R.W Siegel and J.S Dordick, Langmuir 20 6800 (2004).

7. J. Teixeira, J. Appl. Cryst. 21, 781 (1988). 